\title{
Nonequilibrium stationary microstructures in surface chemical reactions
}

\author{
M. Hildebrand, A. S. Mikhailov, and G. Ertl \\ Fritz-Haber-Institut der Max-Planck-Gesellschaft, Faradayweg 4-6, D-14195 Berlin, Germany
}

(Received 14 July 1998)

\begin{abstract}
Adsorbates with attractive lateral interactions between molecules in the presence of adsorption and thermal desorption are considered. We show that a sufficiently strong nonequilibrium reaction or the process of photoinduced desorption can destabilize uniform stationary phases of the adsorbates and lead to nonequilibrium microstructures whose wavelength is typically shorter than the diffusion length and may hence be on the micrometer to nanometer scale. Despite their small sizes, such microstructures are not destroyed by internal statistical fluctuations. [S1063-651X(98)03511-9]
\end{abstract}

PACS number(s): 82.20.Mj, 47.54.+r, 68.10.Jy, 82.65.Jv

\section{INTRODUCTION}

Catalytic chemical reactions in adsorbates on metal surfaces are an important and intensively investigated class of nonequilibrium physicochemical systems [1]. Application of photoelectron emission microscopy (PEEM) has led to the discovery of a rich variety of spatiotemporal patterns in these systems, including traveling or rotating spiral waves and turbulence [2]. The characteristic length scales of such patterns lay in the range of tens of micrometers, whereas the diffusion length of the mobile surface species was about $1 \mu \mathrm{m}$ and the lattice constant was of order $10^{-4} \mu \mathrm{m}$. Therefore, all these patterns were effectively macroscopic and their properties could well be described by the classical reaction-diffusion models neglecting fluctuations and interactions between the molecules [3]. The recent development of the fast scanning probe, however, makes it possible to study surface reactions with an atomic resolution [4]. Moreover, the high-resolution PEEM is coming up and would soon allow one to image the reaction patterns on the submicrometer scale.

Nonequilibrium spatiotemporal patterns on scales shorter than the diffusion length may result from the interplay of reactions and attractive potential interactions between adsorbate molecules $[5,6]$. Indeed, short-scale transient patterns develop at the initial stage of the phase separation process $[7,8]$. It is known that nonequilibrium reactions can freeze these transient patterns and thus lead to the emergence of stationary microscopic patterns in binary mixtures, such as polymer blends [9-11]. It has earlier been suggested [12] that adsorption and thermal desorption processes can have similar effects in the adsorbates with attractive interactions between particles. However, our theoretical analysis of such systems [13] has shown that adsorption and thermal desorption do not lead to the development of stationary microstructures. Instead the system always evolves to a state of thermal equilibrium characterized by uniform adsorbate coverage.

Adsorption and thermal desorption correspond to exchange of particles between the surface and a large gas reservoir that form together a closed system. This system must therefore relax to a state of thermal equilibrium that cannot depend on any purely kinetic properties of the system. Consequently, microstructures with the period dependent on the diffusion constants of adsorbed particles are not possible in such closed systems. Adsorption and thermal desorption rep- resent "equilibrium reactions" which are not themselves able to induce the formation of kinetic spatially modulated stationary phases. For polymer blends, such special behavior of equilibrium chemical reactions has been noted in the discussion [14].

Thus, an interesting and previously not addressed question is what would happen if, in addition to adsorption and thermal desorption, the considered system includes an irreversible nonequilibrium surface chemical reaction.

The theoretical analysis of kinetic processes in systems undergoing a first-order phase transition is usually performed in the framework of the Cahn-Hilliard equation $[15,16]$. This equation represents a local approximation that is valid when the radius of interactions between the particles is much shorter than all characteristic length scales of emerging spatial patterns. Interactions between adsorbed molecules on metal surfaces are often mediated through the substrate by chemisorption-induced modulation of the local electronic structure [17] or by chemisorption-induced elastic stresses [18] and may therefore extend over relatively long distances. On the other hand, the characteristic diffusion lengths of some adsorbed species may be relatively short and therefore not strongly different from the radius of interactions between adsorbed particles. In such situations, the approaches based on the Cahn-Hilliard equation are not applicable and a nonlocal kinetic description is needed.

We have recently proposed [5] and then directly derived from the underlying microscopic master equation [13] a nonlocal mesoscopic kinetic equation for the adsorbates that explicitly takes into account lateral potential interactions between the particles. We have also shown how internal fluctuations can be incorporated into this description by adding internal noises into the nonlocal kinetic equation [13]. The small parameter used in the derivation is the inverse number of surface lattice sites in the area with the interaction radius. Hence, this mean-field kinetic equation with fluctuations is generally applicable when attractive interactions extend over many lattice lengths. A similar nonlocal equation without fluctuating terms has recently been independently constructed for binary alloys in the limit of long-range interactions [19]. The novel mesoscopic nonlocal kinetic equation has already been used [13] to describe the nucleation kinetics of equilibrium phase transitions in adsorbates in the presence 
of adsorption and desorption processes far from the critical point of the phase transition.

The aim of this study is to theoretically investigate the influence of irreversible nonequilibrium surface chemical reactions on phase transitions in adsorbates in the presence of adsorption and thermal desorption processes. We show that in these systems strong enough nonequilibrium reactions can induce the formation of stationary microstructures with the wavelengths lying in the submicrometer and nanoscale range. Our analysis is based on the mesoscopic kinetic equation. This allows us to study the effects of interactions with a finite radius and investigate the influence of fluctuations in such reactive adsorbates.

In Sec. II we formulate a mesoscopic kinetic equation for the considered systems and compare this approach with other kinetic descriptions based on the Cahn-Hilliard equation. The linear stability analysis of the uniform adsorbate phases is performed in Sec. III. Its results are used to construct bifurcation diagrams for the considered system and discuss the parameter dependence of the critical wavelength. The nonlinear stage of the instability and the morphology of the developing stationary microstructures are then investigated by numerical simulations in Sec. IV. In Sec. V possible experimental realizations of the considered nonequilibrium microstructures are discussed.

\section{THE MESOSCOPIC KINETIC EQUATION}

As an example, we consider a system with a single kind of molecule that adsorbs on, desorbs from, and diffusively moves across a solid surface. These molecules exhibit pairwise attractive interactions and are subject to a first-order nonequilibrium chemical reaction whose product immediately leaves the surface. This system is described (see [13]) by the following mesoscopic kinetic equation for the local coverage $c$ of adsorbed molecules:

$$
\begin{aligned}
\frac{\partial c}{\partial t}= & k_{a} p_{0}(1-c)-k_{d} c-k_{r} c+\frac{\partial}{\partial \mathbf{r}}\left(\frac{D}{k_{B} T}(1-c) c \frac{\partial U(\mathbf{r})}{\partial \mathbf{r}}\right) \\
& +D \frac{\partial^{2} c}{\partial \mathbf{r}^{2}}+\xi(\mathbf{r}, \mathbf{t}) .
\end{aligned}
$$

The first term describes the rate of adsorption determined by the initial sticking coefficient $k_{a}$, the partial pressure of the gaseous species $p_{0}$, and the fraction of the surface being adsorbate-free, $1-c$. In the following terms, $k_{d}$ is the desorption rate constant, $k_{r}$ is the reaction rate constant, $D$ is the molecular diffusion constant for the adsorbate, and $T$ is the temperature. The local surface potential $U(\mathbf{r})$, experienced by adsorbed molecules, is caused by lateral pairwise attractive interactions and is given by the integral

$$
U(\mathbf{r})=-\int u\left(\mathbf{r}-\mathbf{r}^{\prime}\right) c\left(\mathbf{r}^{\prime}\right) d \mathbf{r}^{\prime}
$$

The rate constant for desorption can then be written as

$$
k_{d}=k_{d, 0} \exp \left[U(\mathbf{r}) / k_{B} T\right]
$$

where $k_{d, 0}$ is its value in the absence of interactions.

Equation (1) includes a term that describes viscous flow of the adsorbate induced by the potential gradient. As a simple approximation for the interaction potential, we choose the Gaussian profile

$$
u(r)=\left(u_{0} / \pi r_{0}^{2}\right) \exp \left(-r^{2} / r_{0}^{2}\right)
$$

where $u_{0}$ specifies the interaction strength and $r_{0}$ is the interaction radius.

The first-order reaction model underlying Eq. (1) can actually represent a bimolecular reaction where the second species reacts directly from the gas phase (Eley-Rideal mechanism). Another possible realization of this reaction is a process of photoinduced desorption (see further discussion in Sec. V). In both cases the reaction is nonequilibrium since energy is brought with photons or molecules of the second species that hit the surface. Therefore, the reaction rate constant $k_{r}$ does not depend, in contrast to the thermal desorption rate constant, on the local potential for the adsorbed particles.

The random term $\xi(\mathbf{r}, t)$ in Eq. (1) combines internal noises of reaction, adsorption, desorption, and diffusion processes:

$$
\begin{aligned}
\xi(\mathbf{r}, t)= & \frac{1}{Z^{1 / 2}} \sqrt{k_{a} p_{0}(1-c)} f_{a}(\mathbf{r}, t)+\frac{1}{Z^{1 / 2}} \sqrt{k_{d, 0} c} \exp \left(U(\mathbf{r}) / 2 k_{B} T\right) f_{d}(\mathbf{r}, t)+\frac{1}{Z^{1 / 2}} \sqrt{k_{r} c} f_{r}(\mathbf{r}, t) \\
& +\frac{1}{Z^{1 / 2}} \frac{\partial}{\partial \mathbf{r}}\{\sqrt{2 D c(1-c)} \mathbf{f}(\mathbf{r}, t)\},
\end{aligned}
$$

where the random forces $f_{a}(\mathbf{r}, t), f_{d}(\mathbf{r}, t), f_{r}(\mathbf{r}, t)$, and $\mathbf{f}(\mathbf{r}, t)$ represent independent white noises of unit intensity, so that $\left\langle f_{a}(\mathbf{r}, t) f_{a}\left(\mathbf{r}^{\prime}, t^{\prime}\right)\right\rangle=\left\langle f_{d}(\mathbf{r}, t) f_{d}\left(\mathbf{r}^{\prime}, t^{\prime}\right)\right\rangle=\left\langle f_{r}(\mathbf{r}, t) f_{r}\left(\mathbf{r}^{\prime}, t^{\prime}\right)\right\rangle$ $=\left\langle f_{x}(\mathbf{r}, t) f_{x}\left(\mathbf{r}^{\prime}, t^{\prime}\right)\right\rangle=\left\langle f_{y}(\mathbf{r}, t) f_{y}\left(\mathbf{r}^{\prime}, t^{\prime}\right)\right\rangle=\delta\left(\mathbf{r}-\mathbf{r}^{\prime}\right) \delta\left(t-t^{\prime}\right)$ and $\left\langle f_{x} f_{y}\right\rangle=0$. The derivation of the stochastic mesoscopic equation (1) with internal noises (5) from the respective full microscopic master equation on a lattice, employing partial averaging (coarse graining), has been performed in Ref. [13] (see also the general discussion of mesoscopic stochastic equations [20,21]). The parameter $Z$ in Eq. (5) is given by the number of lattice sites per unit surface area, so that the atomic lattice length is $l_{0}=Z^{-1 / 2}$. A small parameter employed in this derivation is $\delta=\left(r_{0}^{2} Z\right)^{-1}$, i.e., the inverse number of lattice sites inside an area of the interaction ra- 

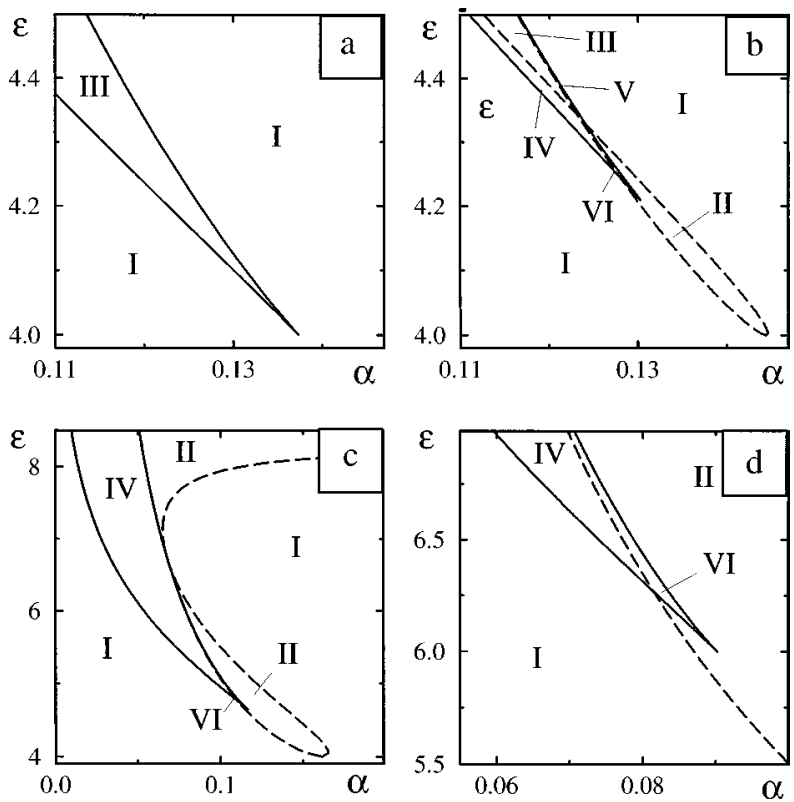

FIG. 1. Bifurcation diagrams in the parameter plane $(\alpha, \varepsilon)$ for (a) $\beta=0$ (no reaction), (b) $\beta=0.05$, (c) $\beta=0.162$, and (d) $\beta=0.5$. The dashed line shows the instability boundaries with respect to periodic spatial modulation in the limit $r_{0} \ll L_{r}$. In the marked regions the system has (I) single uniform phase, (II) single spatially modulated phase, (III) two uniform phases, (IV) one uniform low-density and one spatially modulated phase, (V) one uniform high-density and one spatially modulated phase, and (VI) two different stable spatially modulated nonequilibrium phases.

dius. The condition $\delta \ll 1$ implies that $r_{0} \gg l_{0}$, i.e., that the interaction radius $r_{0}$ should be much larger than the atomic lattice length.

In the absence of the reaction, the considered system has an equilibrium first-order phase transition [22]. Then, its stationary uniform states are given by the roots of the equation

$$
\alpha(1-c)=\exp (-\varepsilon c) c
$$

where the notations $\varepsilon=u_{0} / k_{B} T$ and $\alpha=k_{a} p_{0} / k_{d, 0}$ have been introduced. This is the standard mean-field equation of an adsorption isotherm (cf. [22]). Inside the cusp region of the parameter plane [Fig. 1(a)], the system is bistable: both highand low-density uniform adsorbate phases are possible. The critical point of this equilibrium phase transition $c_{\mathrm{cr}}=0.5$ is located at $\varepsilon=4$ and $\ln \alpha=-2$.

Before the analysis of pattern formation phenomena, described by this model, we want to discuss its relation to the other approaches for the description of reacting systems with first-order phase transitions, based on the Cahn-Hilliard equation.

Assuming that the coverage $c(\mathbf{r}, t)$ does not significantly vary within the distances about the interaction radius $r_{0}$ and neglecting fluctuations, the integro-differential equation (1) can be approximated (as shown in Ref. [13]) by the following local kinetic equation:

$$
\begin{aligned}
\frac{\partial c}{\partial t}= & k_{a} p_{0}(1-c)-k_{d, 0} \exp \left(-\frac{u_{0} c}{k_{B} T}\right) c-k_{r} c \\
& +\frac{\partial}{\partial \mathbf{r}}\left(D_{\mathrm{eff}}(c) \frac{\partial c}{\partial \mathbf{r}}\right)-\frac{\partial}{\partial \mathbf{r}}\left(G(c) \frac{\partial^{3} c}{\partial \mathbf{r}^{3}}\right),
\end{aligned}
$$

where

$$
D_{\mathrm{eff}}(c)=D[1-\varepsilon c(1-c)], \quad G(c)=\frac{1}{2} D \varepsilon r_{0}^{2} c(1-c)
$$

It can also be written in the equivalent form

$$
\frac{\partial c}{\partial t}=Q(c)+\frac{\partial}{\partial \mathbf{r}}\left(M(c) \frac{\partial}{\partial \mathbf{r}} \frac{\delta F}{\delta c(\mathbf{r})}\right),
$$

where

$$
\begin{aligned}
F[c]= & \int\left[-\frac{1}{2} \varepsilon c^{2}+c \ln c+(1-c) \ln (1-c)\right. \\
& \left.+\frac{1}{4} \varepsilon r_{0}^{2}\left(\frac{\partial c}{\partial \mathbf{r}}\right)^{2}\right] d \mathbf{r}, \\
Q(c)= & k_{a} p_{0}(1-c)-k_{d, 0} \exp \left(-\frac{u_{0} c}{k_{B} T}\right) c-k_{r} c,
\end{aligned}
$$

and $M(c)=D c(1-c)$.

Equation (9) represents the local Cahn-Hilliard kinetic equation for the considered system, including additional terms to account for adsorption, desorption, and reaction processes. Note that the dependence of the coefficient $M$ on the coverage $c$ can only be neglected if coverage variations in considered patterns are small, i.e., the patterns have small amplitudes.

Near the critical point $\varepsilon=4$ of the equilibrium phase transition, decomposition in powers of small deviations from the critical coverage $c=0.5$ can be performed here. Retaining only the leading terms, this yields the Landau free-energy functional:

$$
\tilde{F}[c]=\frac{1}{4} \int\left[-\frac{1}{2}(\varepsilon-4) \phi^{2}+\frac{4}{3} \phi^{4}+r_{0}^{2}\left(\frac{\partial \phi}{\partial \mathbf{r}}\right)^{2}\right] d \mathbf{r},
$$

where $\phi=c-0.5$. Putting $c=0.5$ in the kinetic coefficient $M(c)$, the approximate Cahn-Hilliard equation in the vicinity of the point $\varepsilon=4$ is thus obtained:

$$
\frac{\partial c}{\partial t}=Q(c)+D \frac{\partial^{2}}{\partial \mathbf{r}^{2}}\left(\frac{\delta \tilde{F}}{\delta c(\mathbf{r})}\right)
$$

The Cahn-Hilliard equation in the form (13) with the Landau free energy (12) has first been applied by Huberman [16] to describe the influence of chemical reactions on systems with phase transitions. The approaches based on the CahnHilliard equation were later employed in theoretical studies on phase separation phenomena in polymer blends with chemical reactions $[9,10]$. The polymer blends were modeled as simple binary mixtures with components $A$ and $B$ and the reactions $A \rightarrow B$ (with the rate constant $\Gamma_{1}$ ) and $A \leftarrow B$ (with the rate constant $\Gamma_{2}$ ) were taken into account. The reaction rate constants were not influenced by interactions between molecules $A$ and $B$, responsible for the phase transition, and hence only nonequilibrium (e.g., optically induced) reactions were considered. Assuming the incompressibility condition $c_{A}+c_{B}=1$, the reaction part of the kinetic equation (13) had in this case the form 


$$
Q(c)=\Gamma_{2}-\left(\Gamma_{1}+\Gamma_{2}\right) c,
$$

where the notation $c=c_{A}$ is employed. The rate constants of the forward and backward reactions were taken equal $\left(\Gamma_{1}\right.$ $=\Gamma_{2}=\Gamma$ ) by Glotzer et al. [9], who have used the CahnHilliard equation in the form (9) with the free-energy functional (10). Motoyama and Ohta [10] have subsequently investigated the nonsymmetric case $\left(\Gamma_{1} \neq \Gamma_{2}\right)$ in the framework of the Cahn-Hilliard approach, using in their numerical simulations a simple model form for the free energy and performing their analytical investigation for the Landau free energy (12). In both these studies the kinetic coefficient $M(c)$ in the Cahn-Hilliard equation (9) was taken constant.

The adsorbates can also be formally viewed as binary mixtures if the adsorbed molecules are considered as particles $A$ and the empty sites as fictitious particles $B$. Comparing Eq. (14) with Eq. (11), we see that the results of the studies $[9,10]$ can also be applied to reactive adsorbates with short interaction radii in the absence of thermal desorption $\left(k_{d, 0}=0\right)$, provided the coverage in the considered patterns is weakly varying around a certain mean level.

Verdasca et al. [12] have used the Cahn-Hilliard equation (13) with the Landau free energy (12) to study adsorbates with first-order phase transitions in the presence of adsorption and associative desorption processes. These processes were taken into account by choosing

$$
Q(c)=k_{a} p_{0}(1-c)-k_{d} c^{2}
$$

where the first term described adsorption and the second term (with a constant coefficient $k_{d}$ ) corresponded to the associative desorption process.

However, if thermal desorption is considered, the desorption rate constant $k_{d}$ must depend on the local potential. Indeed, the particles leaving the surface must overcome a certain potential barrier that is modulated by the local potential due to the interactions between these adsorbed particles. In the subsequent discussion, Verdasca et al. have pointed out [23] that the experimental data for thermal desorption often show deviations from the mean-field results and therefore Eq. (15) with constant coefficients may, perhaps, still be used as a phenomenological description of adsorption and desorption processes.

The choice of an expression for the thermal desorption rate cannot be arbitrary. There are two different situations involving adsorption and thermal desorption. On one hand, these processes can take place in an open reactor. On the other hand, adsorption and thermal desorption can simply correspond to reversible exchange of particles between a surface and a gas reservoir in a closed system. The partial pressure in the gas phase can be constant in both of these situations, if the pumping rate in the open reactor is high or if the gas reservoir is large in the closed system. It is important that both situations are described by exactly the same kinetic equation for the adsorbate and therefore the behavior of the adsorbate should be the same in both cases. But a closed system must relax to the state of thermodynamic equilibrium whose properties cannot depend on any kinetic coefficients, such as the diffusion constant of adsorbed particles on the surface. If, however, the rate of thermal (associative) desorption is phenomenologically chosen in the form (15), the
Cahn-Hilliard equation (13) yields steady states (see [12]) whose properties depend on the diffusion constant and that cannot, therefore, correspond to the true thermal equilibrium.

Apparently, since the Cahn-Hilliard equation is based on the mean-field approximation, it is consistent only with the expressions for the adsorption and desorption rates that are derived in the same mean-field approximation. Indeed, in our study [13] we have shown that if the monomolecular thermal desorption is described by the classical coverage-dependent desorption rate constant $k_{d}=k_{d, 0} \exp \left(-u_{0} c / k_{B} T\right)$ obtained in the mean-field approximation (see, e.g., [22]), the system always relaxes to the state of thermal equilibrium with only uniform adsorbate phases. From our point of view, the theoretical results of Ref. [12] may apply, therefore, only to the situations where the associative desorption is not thermal, but externally induced.

Thus, we see that previous investigations do not yield results applicable in situations where both thermal desorption and nonequilibrium reactions are present and are influencing the adsorbate phase transition. Moreover, since they were based on the Cahn-Hilliard equation, their results are not applicable when the interaction radius is comparable with characteristic length scales of the appearing patterns. Our analysis of the problem is performed in the next sections in the framework of the mesoscopic kinetic equation (1).

\section{LINEAR STABILITY ANALYSIS AND BIFURCATION DIAGRAMS}

To analyze instabilities leading to the formation of nonequilibrium stationary microstructures and to determine bifurcation boundaries in the parameter space of the system, we first consider in this section its deterministic limit, neglecting noise terms in the kinetic equation (1). The stationary uniform states of the system are given by the roots of the equation

$$
\alpha(1-c)-\beta c=\exp (-\varepsilon c) c
$$

where we have additionally introduced the dimensionless reaction rate constant $\beta=k_{r} / k_{a} p_{0}$. Without the reaction $(\beta$ $=0$ ), it reduces to the mean-field equation of an adsorption isotherm that describes a first-order phase transition in the adsorbate [22]. Inside the cusp region, whose boundaries are shown by the bold line in Fig. 1(a), the system is bistable: both dense and dilute uniform adsorbate phases are possible. Introduction of the nonequilibrium monomolecular reaction modifies the phase diagram: As the dimensionless reaction rate constant $\beta$ increases, the cusp shifts to higher values of the dimensionless interaction strength $\varepsilon=u_{0} / k_{B} T$ and smaller values of the dimensionless relative adsorption rate $\alpha=k_{a} p_{0} / k_{d, 0}$ [see Figs. $\left.1(\mathrm{~b})-1(\mathrm{~d})\right]$. The critical point is located at $\varepsilon^{*}=4(1+\beta)$ and $\alpha^{*}=(1+\beta)^{-1} e^{-2}$; the coverage at this point is $c^{*}=[2(1+\beta)]^{-1}$.

The stability of uniform states is tested by adding small perturbations $\delta c(x, t)=\delta c \exp \left(\gamma_{\kappa} t\right) \exp (i \kappa x)$ in the onedimensional system. Linearizing the kinetic equation (1), we find that the increment of growth of these perturbations is given by 


$$
\begin{aligned}
\gamma_{\kappa}= & -k_{a} p_{0}-k_{r}+k_{d, 0}(\varepsilon c-1) e^{-\varepsilon c}-D \kappa^{2}[1-\varepsilon c(1-c)] \\
& -\left[k_{d, 0} \varepsilon c e^{-\varepsilon c}+D \kappa^{2} \varepsilon c(1-c)\right]\left(1-e^{-r_{0}^{2} \kappa^{2} / 4}\right)
\end{aligned}
$$

We see that $\gamma_{\kappa}$ is always real and the unstable modes are not oscillatory. The situation is similar to the classic Turing instability in activator-inhibitor systems: The dispersion $\gamma_{\kappa}$ has a single maximum at a wave number $\kappa_{0}$ which changes its sign at the instability, i.e., the stability boundaries and the wave number $\kappa_{0}$ of the first unstable mode are determined by the conditions that $\gamma_{\kappa}=0$ and $\partial \gamma_{\kappa} / \partial \kappa=0$ at $\kappa=\kappa_{0}$.

Using Eq. (17), the wave number $\kappa_{0}$ of the first unstable spatial mode is determined as

$$
\begin{aligned}
\kappa_{0}^{2}= & \frac{1}{1-c_{0}}\left\{-\frac{1}{L_{d}^{2}}+\frac{1}{2 L_{r}^{2}}\right. \\
& \left.\times\left[-1+\left(1+\frac{16 L_{r}^{2}}{r_{0}^{2}}\left(1-c_{0}\right)\right)^{1 / 2}\right]\right\} .
\end{aligned}
$$

Two different diffusion lengths are introduced here: $L_{d}$ $=\left(D / k_{d, 0}\right)^{1 / 2} \exp \left(\varepsilon c_{0} / 2\right)$ is the diffusion length of adsorbed particles with respect to their thermal desorption, while $L_{r}$ $=\left(D / k_{r}\right)^{1 / 2}$ is their diffusion length with respect to the nonequilibrium first-order chemical reaction. The parameter $c_{0}$ is the coverage in the uniform state at the respective instability boundary.

It follows from Eq. (18) that the wave number $\kappa_{0}$ vanishes and therefore the wavelength $\lambda_{0}=2 \pi / \kappa_{0}$ of the first unstable mode diverges when the interaction radius $r_{0}$ approaches a critical value $r_{c}$ given by equation

$$
r_{c}=2 L_{d}\left(\frac{1-c_{0}}{1+L_{r}^{2} / L_{d}^{2}}\right)^{1 / 2} .
$$

When $r_{0}>r_{c}$, Eq. (18) yields $\kappa_{0}^{2}<0$, implying that the instability is then absent. On the other hand, when the characteristic radius of the lateral interactions is small as compared with both characteristic diffusion lengths, i.e., if the conditions $r_{0} \ll L_{r}$ and $r_{0} \ll L_{d}^{2} / L_{r}$ are satisfied, the wavelength of the first unstable mode can be approximately estimated using Eq. (18) as

$$
\lambda_{0}=2^{1 / 2} \pi\left(1-c_{0}\right)^{1 / 4}\left(r_{0} L_{r}\right)^{1 / 2} .
$$

(This result might also be obtained using the Cahn-Hilliard approximation. It agrees with the respective estimate derived using the Cahn-Hilliard kinetic equation in Ref. [9] for the reactive binary mixtures, such as polymer blends.) Thus, the critical wavelength would generally lie in this limit between the radius $r_{0}$ of lateral interactions and the diffusion length $L_{r}$.

The instability boundaries can easily be determined when the interaction radius $r_{0}$ is much shorter than the wavelength of the first unstable modes. If $r_{0} \kappa \rightarrow 0$, the last term on the right-hand side of Eq. (17) can be neglected and $\gamma_{\kappa}$ is simply given by

$$
\gamma_{\kappa}=\gamma_{\kappa=0}-D \kappa^{2}[1-\varepsilon c(1-c)] .
$$

If a uniform steady state with the coverage $c$ is stable with respect to uniform perturbations, $\gamma_{\kappa=0}=-k_{a} p_{0}-k_{r}$ $+k_{d, 0}(\varepsilon c-1) e^{-\varepsilon c}$ must be negative. This state will, however, be unstable with respect to nonuniform, spatially modulated perturbations with sufficiently large wave numbers $k>\gamma_{\kappa=0} / D_{\text {eff }}(c)$ if the effective diffusion coefficient $D_{\text {eff }}(c)=D[1-\varepsilon c(1-c)]$ is negative. Thus, the instability boundaries are determined in the limit $r_{0} \rightarrow 0$ by the equation $D_{\text {eff }}(c)=0$ together with Eq. (16). Note that the solution of $D_{\text {eff }}(c)=0$ yields then the coverage $c_{0}$ at the respective instability boundary that should be substituted into Eq. (20).

The computed instability boundaries in the parameter plane $(\alpha, \varepsilon)$ at different values of the dimensionless relative reaction rate constant $\beta=k_{r} / k_{a} p_{0}$ in the limit $r_{0} \rightarrow 0$ are shown in Fig. 1. In the absence of the reaction $(\beta=0)$ the system has one stable uniform state in the region (I) and two uniform stable states in the region III inside the cusp [Fig. $1(\mathrm{a})]$.

As $\beta$ is increased [Fig. 1(b)], the instability first develops at the cusp boundaries (in the regions IV and V) and near the critical point (in the region II). The system remains, however, in the stable uniform phase in region I and has two stable uniform phases in the region III. Note that in the region IV the system has a stable uniform low-density phase, whereas the uniform high-density phase is unstable with respect to nonuniform perturbations. The situation is opposite in the very narrow region $\mathrm{V}$, where the uniform high-density phase is stable and the uniform low-density phase is unstable and develops spatial modulation. In region VI both uniform phases are unstable with respect to periodic spatial modulation.

When the reaction rate is increased, the bifurcation diagram undergoes a significant change [Fig. 1(c)]. The region $\mathrm{V}$ disappears and the region II extends to the domain of high values of the parameter $\varepsilon$, specifying the relative intensity of attractive lateral interactions. If the reaction is further increased, the dashed boundary of the region II moves in the right direction and we have eventually a situation where the instability with respect to spatial modulation is present in a large part of the parameter plane [Fig. 1(d)], even far from the cusp region.

As noted in the Introduction, the radius of lateral interactions in adsorbates can be relatively large, especially if these interactions are mediated through elastic deformations in the metal substrate. Therefore, evolution of the bifurcation diagrams under an increase in the interaction radius should be investigated. To illustrate the effects of nonlocal interactions, we show how typical bifurcation diagrams in the parameter plane $(\beta, \varepsilon)$ are modified when the interaction radius is increased.

Let us first consider, as a simple example, the system in the absence of thermal desorption $\left(k_{d, 0}=0\right)$. Figure 2 shows in this case the family of the instability boundaries of the uniform state in the plane $(\beta, \varepsilon)$ for different values of the dimensionless radius $\rho_{0}=r_{0} / L_{r}$. The uniform state is unstable with respect to periodic spatial modulations inside the regions bounded by these curves. The lowest curve corresponds to the limit $\rho_{0} \rightarrow 0$. As the interaction radius is increased, the instability region moves upwards to higher values of the effective interaction strength $\varepsilon$. Therefore, if we fix $\varepsilon$ and increase $\rho_{0}$, the interval of $\beta$ values where the uniform phase is unstable will shrink until at a critical value of $\rho_{0}$ the two instability boundaries merge and for larger $\rho_{0}$ 


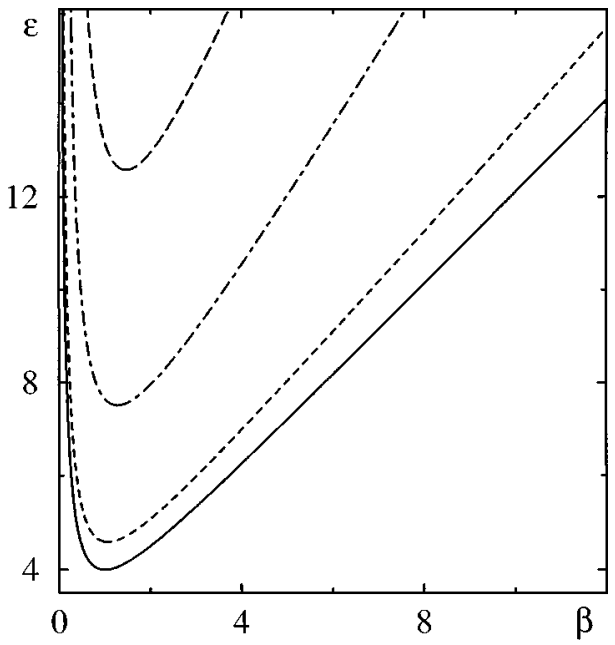

FIG. 2. Bifurcation diagram in the parameter plane $(\beta, \varepsilon)$ in the absence of thermal desorption $\left(k_{d, 0}=0\right)$ for several different values of the dimensionless interaction radius: $\rho_{0}=0$ (solid curve), $\rho_{0}$ $=0.1$ (dashed), $\rho_{0}=0.5$ (dot-dashed), and $\rho_{0}=1.0$ (long-dashed curve). Inside the regions bounded by these curves, a single, spatially modulated phase is found at the respective values of $\rho_{0}$. Outside of these regions the system has a single stable uniform phase.

the instability is absent for any values of $\beta$. This follows from the fact that the nonlocal terms contributing to the growth rate in Eq. (17) all have negative signs, i.e., they have a stabilizing effect on the uniform phase.

In the presence of strong enough thermal desorption, the situation is more complex. When the desorption rate constant exceeds $k_{d, 0}=e^{2} k_{r}$, the system can have two coexisting uniform phases. In the plane $(\beta, \varepsilon)$ the critical point of this transition is located at $\beta^{*}=\left[e^{-2} k_{d, 0} / k_{r}-1\right]^{-1}$ and $\varepsilon^{*}=4\left(1+\beta^{*}\right)$. Figure 3 shows bifurcation diagrams in this plane for $k_{d, 0}=50 k_{r}$ and different values of the dimensionless interaction radius $\rho_{0}$. Similar to the diagrams shown in Fig. 1, they include the cusp region where the system has two uniform states stable with respect to uniform perturbations. The boundaries of this region are determined by Eq. (16) and do not depend on the interaction radius. However, the boundaries of the instabilities with respect to nonuniform perturbations (shown by dashed curves in Fig. 3) are sensitive to variations of the interaction radius. Together with the cusp, these boundaries determine regions I to VI, which are labeled here in the same way as in Fig. 1.

When the interaction radius is very small (i.e., $\rho_{0} \rightarrow 0$ ), the diagram [Fig. 3(a)] includes a relatively wide region II where the system has only one spatially modulated phase. Additionally, however, inside the cusp regions IV and VI are found where the system has two uniform phases and either one or both of them are unstable with respect to the periodic modulation. If we now start to increase the interaction radius, the instability boundaries (i.e., the dashed curves) begin to move in the directions indicated by arrows in Fig. 3. When $\rho_{0}=0.1$ [Fig. 3(b)], the unstable region II shifts upwards and becomes more narrow (cf. Fig. 2). Moreover, inside the cusp region the stability boundary of the low-density uniform phase nearly merges with its existence boundary, so that region VI has almost disappeared. At $\rho_{0}=0.17$ [Fig. 3(c)], regions II and VI are already absent. Furthermore, since the stability boundary for the high-density phase (the dashed curve) has moved towards its existence boundary (the right solid curve), a new region III has appeared. Finally, upon further increasing $\rho_{0}$, the unstable region IV of the dense phase shrinks until it eventually also disappears [cf. Fig. 3(d) with $\left.\rho_{0}=0.23\right]$.

The evolution of the stationary microstructures under an increase of the interaction radius can also be followed by looking at the respective dependence of the wavelength $\lambda_{0}$ of the first unstable mode, generally determined by Eq. (18). When the effective interaction strength is chosen below the critical cusp point (i.e., $\varepsilon<\varepsilon^{*}$ ), we have two instability boundaries and, therefore, two different wavelengths. The typical dependence of these wavelengths on the dimensionless interaction radius is shown in Fig. 4(a). We see that, as a certain critical interaction radius (corresponding to merging of these two boundaries and disappearance of the instability at a given $\varepsilon$ ) is approached, the wavelengths of the two unstable modes come closer and meet while remaining finite. It should be noted that the critical value of $\rho_{0}$ corresponding to the merging point is always smaller than its critical value for the divergence of the characteristic wavelength given by Eq. (19).

The development is different when the parameter $\varepsilon$ is fixed above the critical point (i.e., $\varepsilon>\varepsilon^{*}$ ) [Fig. 4(b)]. In this case, only a single stability boundary (and hence a single wavelength of the first unstable mode) is associated with each of the uniform phases. These wavelengths increase with the interaction radius for both phases. They diverge, going to infinity as $\lambda \propto\left(\rho_{c}-\rho_{0}\right)^{1 / 2}$, when the dimensionless interaction radius reaches the critical values $\rho_{c}$ given by Eq. (19). Thus, close to $\rho_{c}$ the appearing modulated structures would represent macroscopic patterns. Note that the divergence of $\lambda$ takes place when the stability boundary for a given uniform phase hits the corresponding existence boundary. Therefore the value of $c_{0}$ in Eq. (19) for the critical interaction radius is

$$
c_{0}=\frac{1}{2(1+\beta)}\left[1 \pm\left(1-\frac{4(1+\beta)}{\varepsilon}\right)^{1 / 2}\right]
$$

Here the signs + and - correspond to the low- and highdensity uniform phases, respectively.

We have shown additionally by thin solid and dashed curves in Figs. 4(a) and 4(b) the dependences of the critical wavelengths yielded by the approximation (20) that is equivalent to using the Cahn-Hilliard kinetic equation. Generally, this approximation is good only for very small interaction radii. However, as seen from Fig. 4(a), it correctly yields the wavelength of the first unstable mode below the critical cusp point at lower reaction rate constants almost up to the moment when the two boundaries merge and the instability disappears at a critical interaction radius.

Our bifurcation diagrams have been constructed in this section by a linear stability analysis of the uniform steady states. Therefore, they only show the boundaries where these uniform states become unstable and the growth of spatially nonuniform modes begins. The nonlinear stage of such instabilities and the properties of developing nonlinear stationary patterns are numerically analyzed in Sec. IV. 

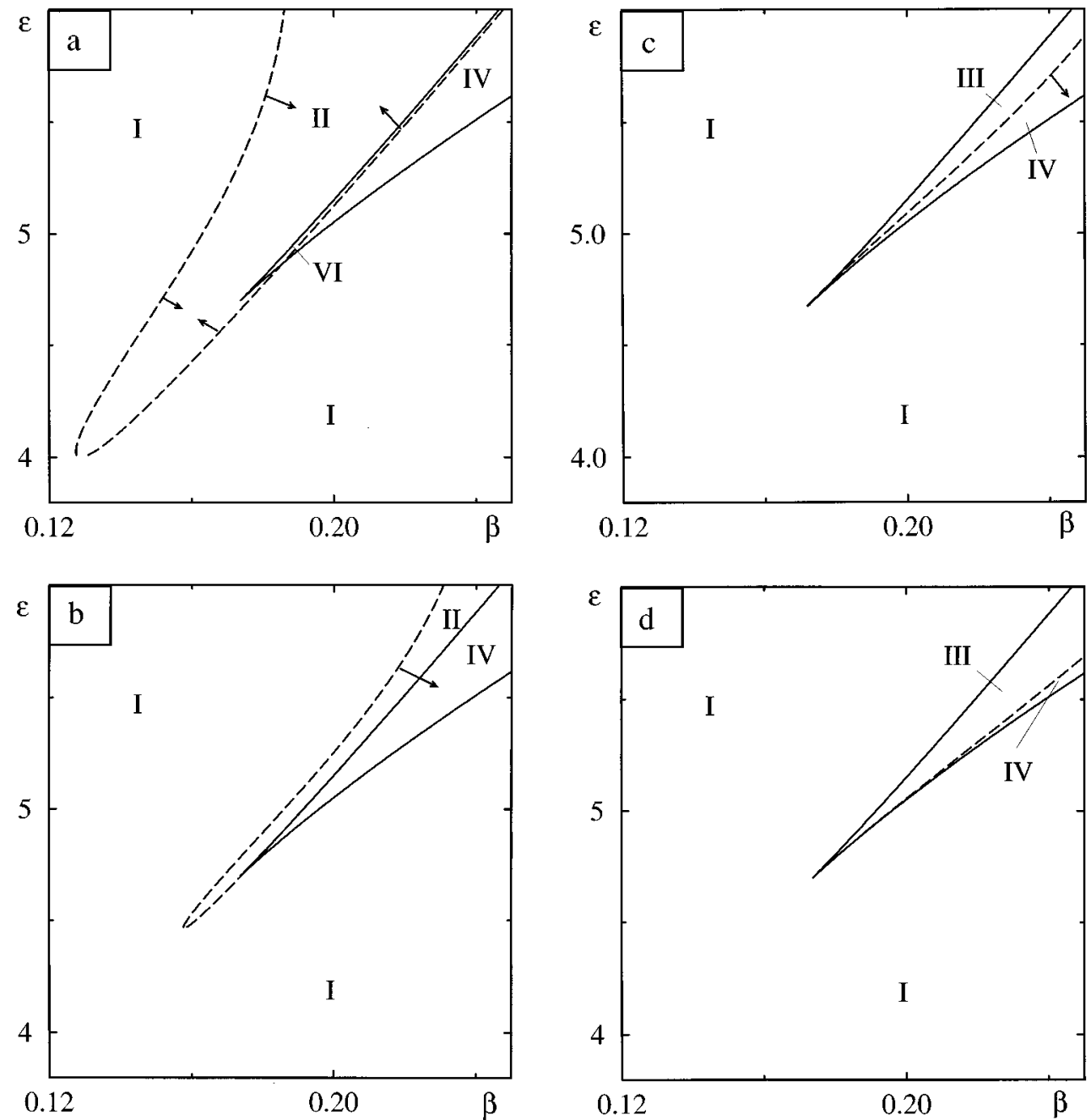

FIG. 3. Bifurcation diagrams in the parameter plane $(\beta, \varepsilon)$ for $k_{d, 0} / k_{r}=50$. The solid curves show the boundaries of the region where two uniform states, both stable with respect to uniform perturbations, are present. The instability boundaries of uniform phases with respect to periodic spatial modulation are shown by dashed curves at several different values of the dimensionless interaction radius: (a) $\rho_{0} \rightarrow 0$, (b) $\rho_{0}=0.1$, (c) $\rho_{0}=0.17$, and (d) $\rho_{0}=0.23$.

\section{NUMERICAL SIMULATIONS}

The behavior of the system has been followed in numerical simulations. In the one-dimensional system, we have found that the instability always leads to stationary periodic structures. Figure 5 gives two characteristic examples of these patterns. The structure shown in Fig. 5(a) has a small amplitude and its profile is almost harmonical. It can therefore be described using the local Cahn-Hilliard approximation. In contrast to this, the structure shown in Fig. 5(b) is characterized by two different characteristic length scales and can be viewed as a periodic array of domains with sharp interfaces. The spatial period of this structure is much larger than the interaction radius. However, the width of the domain boundaries is in this case close to the interaction radius. Moreover, the adsorbate coverage changes significantly across the structure. Even for short interaction radii, it can therefore correctly be described only using the nonlocal kinetic equation (1). Note that small-amplitude structures have been found in our simulations only in narrow parameter regions. Typically, the coverage profiles in the developing microstructures show a large variation and these profiles are strongly nonharmonical.
In two-dimensional systems, the instabilities lead to the development of spatial patterns that tend to approach a periodic spot array or an irregular labyrinthine structure. The final stage of such an evolution is very slow when the system parameters are chosen in the middle of the unstable region. Therefore, in our numerical simulations we were not able to reach the asymptotic periodic states in the two-dimensional systems. However, our simulation results already allow us to show the principal morphologies of the emerging patterns.

When the interaction strength $\varepsilon$ is gradually increased while keeping the parameters $\alpha$ and $\beta$ constant, morphological transitions from a dense-on-dilute spot array [Fig. 6(a)] via complex labyrinthine phases [Fig. 6(b)] towards a diluteon-dense spot array [Fig. 6(c)] are observed in region II of the phase diagram in Fig. 1(d).

Inside the cusp regions in Fig. 1, two uniform states corresponding to high and low coverages are possible. Usually the considered instability develops only for one of these states, while the second remains stable with respect to small perturbations. There are, however, narrow parameter regions (denoted as VI in Figs. 1 and 3) where both uniform states are unstable. Different initial conditions can lead here to two 

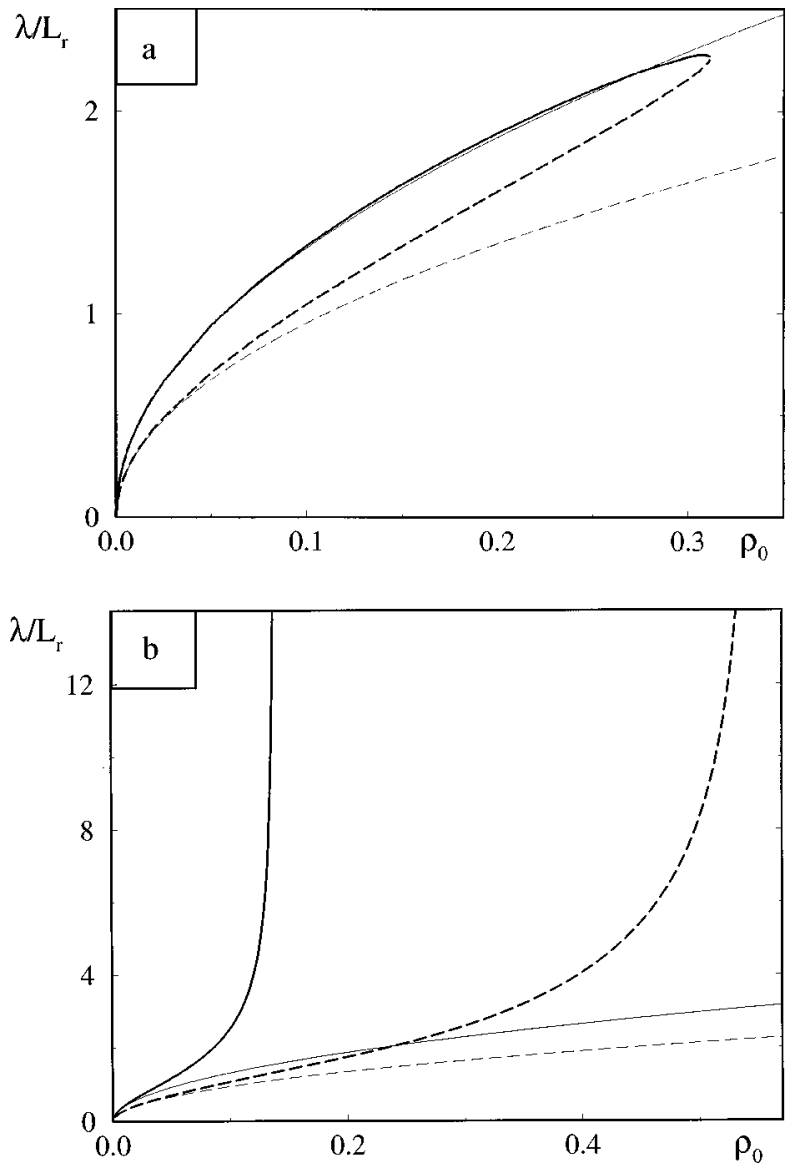

FIG. 4. Dependence of the wavelength of the first unstable mode $\lambda_{0}$ on the dimensionless interaction radius $\rho_{0}$ for $\varepsilon=6$ and (a) $k_{d, 0} / k_{r}=1$ and (b) $k_{d, 0} / k_{r}=50$ (b). The thin lines show the respective dependence yielded by the approximation (20).

different kinds of modulated phases. Preparing an initial condition where half of the system is covered by the spot array and the other half with the labyrinthine phase, we find [Fig. $6(d)]$ that in this case the interface separating the two spatially modulated phases slowly moves into the region filled with the labyrinthine array.

The total size of the system shown in Fig. 6 is only about three diffusion lengths with respect to the reaction. The characteristic length scale of the patterns would become even shorter if the interaction radius is further reduced [cf. Eq. (20)]. On these microscales, the stochastic nature of the diffusion and reaction processes is significant.
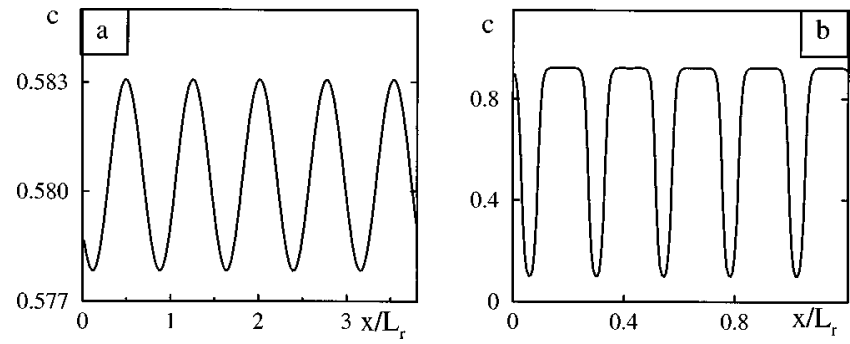

FIG. 5. Coverage profiles of stationary microstructures in a onedimensional system for (a) $\alpha=0.3846, \beta=0.52, \varepsilon=6, \rho_{0}$ $=0.0447$, and $L=3.8 L_{r}$ and (b) $\alpha=0.1, \beta=0.2, \varepsilon=6, \rho_{0}$ $=0.0141$, and $L=1.15 L_{r}$.

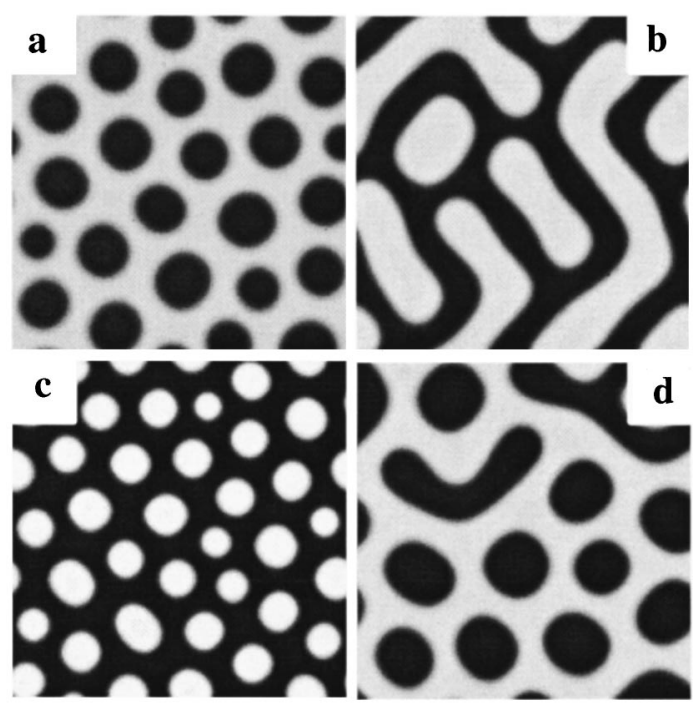

FIG. 6. Typical reaction-induced microstructures in twodimensional systems. The darker areas correspond to regions with higher adsorbate coverages. In Figs. 2(a)-2(c) the total size of the system is $L=3.39 L_{r}$, the dimensionless interaction radius is $\rho_{0}$ $=0.042$, the dimensionless rate constant of thermal desorption is $\alpha=0.09$, the dimensionless reaction rate constant is $\beta=0.5$, and the dimensionless interaction strength is $\varepsilon=6$ (a), $\varepsilon=6.02$ (b), $\varepsilon=8$ (c). In Fig. 2(d) we have $L=3.21 L_{r}, r_{0}=0.04 L_{r}, \alpha=0.0806, \beta$ $=0.5$, and $\varepsilon=6.4$.

The full mesoscopic kinetic equation (1) contains internal noise terms, explicitly taking into account all statistical fluctuations in the system with characteristic lengths exceeding the coarse-graining length that has been used in its derivation (the coarse-graining is performed over surface areas of size less than the interaction radius but still including a relatively high number of lattice sites). Hence, by integration of this partial stochastic integro-differential equation, the influence of statistical fluctuations on the considered microstructures can be directly investigated.

In the deterministic mean-field limit, the properties of the adsorbate patterns do not depend on their absolute sizes with respect to the lattice length $l_{0}$. Indeed, this microscopic length does not enter into the kinetic evolution equation (1) when the noise terms are neglected. When, however, internal noises of the reaction, adsorption, desorption, and diffusion processes are taken into account, their intensities are proportional to the lattice length, as can be seen from Eq. (5). Hence, the noise effects get stronger if, while keeping constant all other parameters and characteristic lengths, we increase the lattice length $l_{0}$. This has a simple explanation. If the lattice length is larger, a smaller number of lattice adsorption sites per characteristic wavelength $\lambda_{0}$ of the developing microstructures is actually found. This means that the individual patterns, such as spots or curved stripes in Fig. 6, would consist of less adsorbed particles and therefore the fluctuations would be stronger.

We have integrated the stochastic differential equation (1) with the noises (5) by discretizing the two-dimensional system over a grid of $160 \times 160$ points. At each grid point, noises were introduced by using independent random number generators. Figure 7 shows typical results of a simulation including the internal noises. In this simulation we have used 


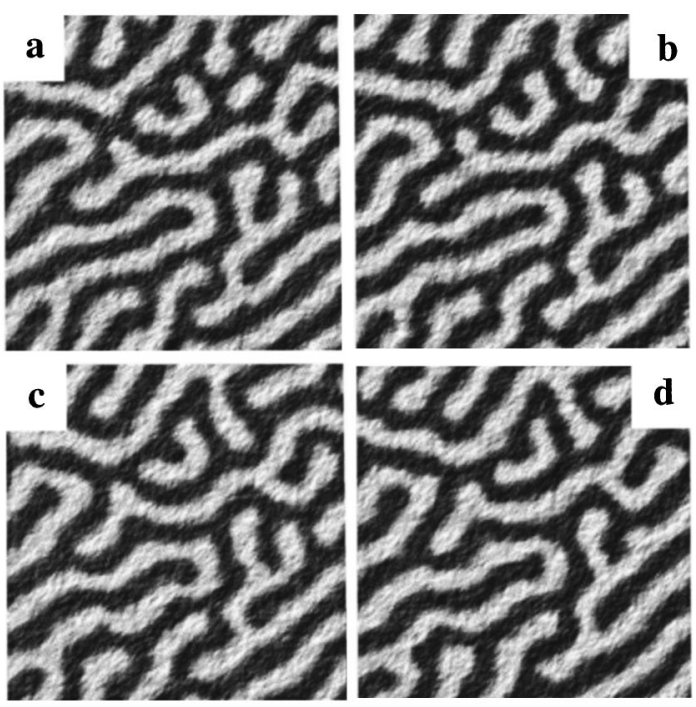

FIG. 7. Fluctuating coverage distributions obtained by integration of the mesoscopic kinetic equation with internal noises for $Z$ $=2.2 \times 10^{3} L_{r}^{-2}$. The time intervals between subsequent frames are $\Delta t=15 / k_{d, 0}$. Other parameters and notations are the same as in Fig. 6(b).

the same system parameters as in the deterministic simulation, whose results are displayed in Fig. 6(b). The additional large parameter $Z$ was taken here equal to $Z=2.2$ $\times 10^{3} L_{r}^{-2}$.

Since $Z=l_{0}^{-2}$, the diffusion length with respect to reaction $L_{r}$ can in this case be expressed in terms of the microscopic lattice length $l_{0}$ as $L_{r} \approx 149 l_{0}$. Furthermore, we can express in terms of the lattice length other characteristic lengths of the considered system, whose values are given in the explanation to Fig. 6(b). The total size of the considered system is $L \approx 506$ lattice lengths and the interaction radius is $r_{0} \approx 6$ lattice lengths. Note that lattice constants for the metals, such as Pt, are usually close to an angstrom. Hence, the total size of the system shown in Fig. 7 can be estimated as only about 50 nanometers. The four frames in Fig. 7 display the spatial distribution of the adsorbate in the same system at three subsequent moments separated by equal time intervals $\Delta t=15 / k_{d, 0}$. We see that, though the fluctuations are relatively strong, they do not destroy the basic morphology of the nonequilibrium microstructure.

\section{DISCUSSION AND CONCLUSIONS}

When possible experiments are considered, the easiest choice of a nonequilibrium first-order reaction, needed to observe the considered microstructures, would be the photodesorption process. Indeed, this process can be viewed as a reaction $X+\nu \rightarrow \star$ where an adsorbed particle $X$ interacts with the photon $\nu$ to produce a vacant surface site $\star$. This reaction has an effective first order. The rate constant of such a reaction does not depend on the surface potential caused by lateral interactions between adsorbed particles if the photon energy is significantly higher than local variations in this potential.

The system parameters that can be varied in such an experiment are the temperature (and hence the dimensionless interaction strength parameter $\varepsilon$ that is inversely proportional

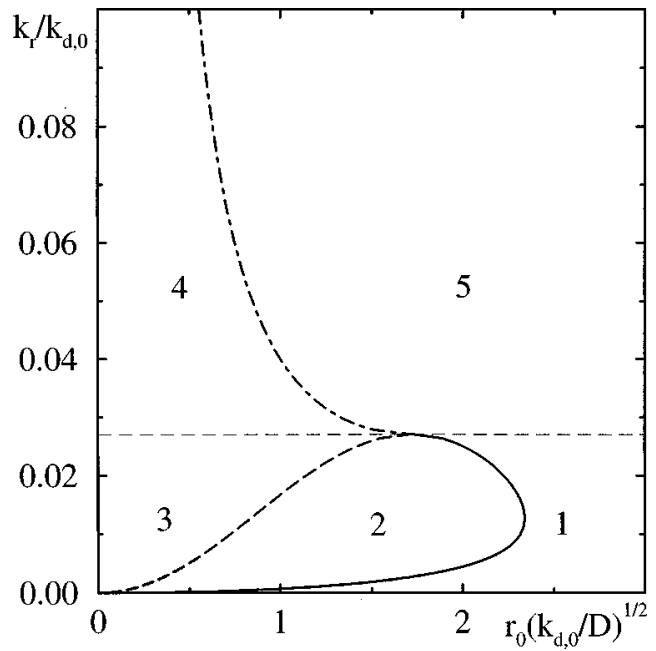

FIG. 8. The diagram showing the regions where, by appropriate tuning of the partial pressure, stationary spatially modulated phases can be found at $\varepsilon=5$. See further explanations in the text.

to the temperature), the light intensity (controlling the rate constant $k_{r}$ ), and the partial pressure $p_{0}$ determining the adsorption rate. To plan an experiment, the existence regions of microstructures in terms of these system parameters should be discussed. Moreover, since the interaction radius $r_{0}$ is usually not well known, it would also be helpful to consider how these regions depend on the variation of the interaction radius.

First we fix the temperature and consider how the behavior of the system is influenced by variation of the photodesorption rate constant $k_{r}$. An important question is now whether, by adjusting the partial pressure, we would be able to enter a region where microstructures are expected. Figure 8 shows boundaries of the regions where various microstructured phases may be observed if the partial pressure is appropriately tuned.

A single or two stable uniform phases can be found in region 1 . In region 2 the high-density uniform phase may be made unstable with respect to spatial modulation by an appropriate choice of the partial pressure, whereas the lowdensity uniform phase is always stable. In region 3 both uniform phases may be unstable and give rise to two different kinds of microstructures. In region 4 the system has only a single phase, which can be nonuniform if the partial pressure is chosen appropriately. In region 5 the system has one stable uniform phase at any partial pressure.

When the interaction radius $r_{0}$ is fixed, three different scenarios take place as the light intensity, controlling the rate constant $k_{r}$, is increased. If the interaction radius exceeds the maximal radius $r_{\max }$, the system has only uniform phases (regions 1 and 5). If it is, however, smaller than $r_{\max }$, microstructures can be found if the photodesorption rate is sufficiently strong as compared with the rate of thermal desorption. As the light intensity, controlling the rate constant $k_{r}$, is increased, microstructures first develop from the highdensity adsorbate phase (region 2).

The subsequent evolution of the system upon further increasing the rate constant $k_{r}$ depends on the magnitude of the interaction radius. At relatively large interaction radii (close to but still smaller than $r_{\max }$ ) the system returns for higher light intensities to region 1 where only uniform phases exist. 


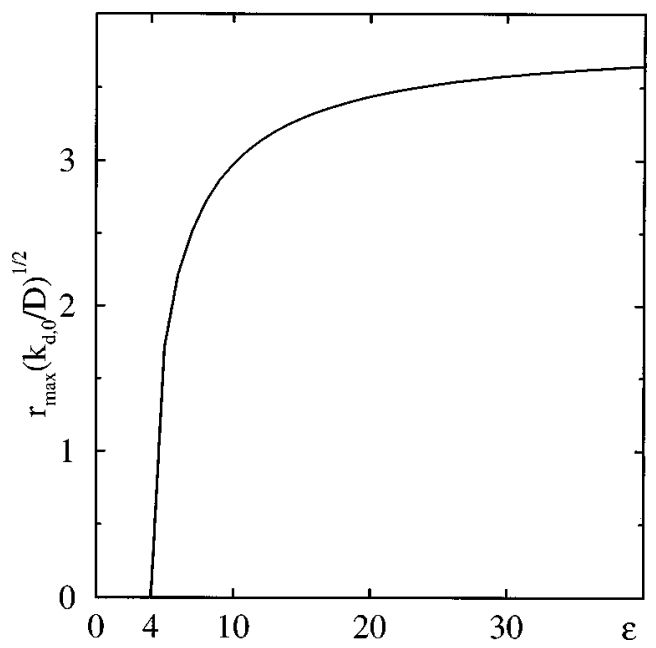

FIG. 9. Dependence of the dimensionless maximum interaction radius $r_{\max }\left(k_{d, 0} / D\right)^{1 / 2}$, needed for the observation of microstructured phases, on the dimensionless interaction strength $\varepsilon$.

However, for even shorter interaction radii the system first goes from region 2 into region 3 . When $k_{r}$ exceeds the threshold value denoted by the thin dashed line in Fig. 8, it enters region 4 with a single spatially modulated phase. In this latter region, the behavior of the considered system is not significantly different from that of the system in the absence of thermal desorption. Note that though Fig. 8 corresponds to the specific choice $\varepsilon=5$, the diagram remains qualitatively unchanged for other choices of this dimensionless interaction parameter.

The maximal interaction radius $r_{\max }$, at which the spatially modulated phases are possible, depends on the rate constant of thermal desorption $k_{d, 0}$ and the dimensionless interaction strength $\varepsilon=u_{0} / k_{B} T$. Figure 9 shows the dependence of the combination $r_{\max }\left(k_{d, 0} / D\right)^{1 / 2}$ on the parameter $\varepsilon$. We see that $r_{\max }$ becomes shorter for smaller $\varepsilon$ (at a fixed thermal desorption rate) and vanishes at $\varepsilon=4$.

The bifurcation diagrams have been constructed in this paper using the linear stability analysis of the uniform states. Therefore, they can only indicate where the respective uniform states should become unstable and stationary spatial modulation should develop. The nonlinear evolution of such an instability has been followed in numerical simulations. In the one-dimensional case, formation of stationary periodic structures was always found above the instability point. For two-dimensional systems, our simulations give evidence of a rich morphology of developing nonequilibrium stationary microstructures. The typical observed patterns represented various spot arrays and complex labyrinthine phases. The interfaces separating regions with low and high adsorbate densities in these patterns have typically the width of the interaction radius. When the system has two different nonequilibrium microstructured phases, nonuniform distributions formed by large patches of these two different phases can be formed on the surface. The boundaries separating these patches slowly move over the surface, until the less favored microstructured phase is completely eliminated.

A detailed study of nonlinear pattern selection was not a purpose of this work. Generally, it is known that pattern selection is controlled by interactions between various growing unstable modes (see, e.g., [24]). They should determine the form of the patterns that develop in the system. Moreover, such nonlinear interactions can make the considered bifurcations subcritical, so that they would be characterized by a hysteresis. We have indeed observed such subcritical behavior in our simulations.

Since the mesoscopic kinetic equation includes internal noises, whose functional form and intensity have been directly determined from the underlying stochastic master equation of the problem, we were also able to consider the influence of fluctuations on the studied microstructures. It was found that, even when the attractive interactions extend only over a relatively small number of lattice neighbors and the internal fluctuations are rather strong, they still do not destroy the basic morphology of the nonequilibrium microstructures.

We want to note that the reaction-induced microstructures, discussed in this paper, together with similar structures in the reactive polymer systems represent, from the physical point of view, a special class of patterns. Indeed, periodic stationary Turing patterns in reaction-diffusion systems have a purely kinetic origin and their wavelength is determined by a combination of diffusion lengths for the reacting activator and inhibitor species [25]. On the other hand, equilibrium spatially modulated phases (see the review [26]) emerge because of the competition between attractive short-range potential interactions and repulsive long-range potential interactions in the system. Their wavelength is therefore determined only by the energetic parameters and does not depend on any kinetic coefficients, such as the diffusion constant. The microstructures studied in this paper are, however, produced by a competition between attractive potential interactions between particles and the kinetic processes (i.e., diffusion and reaction) in the system. This difference is then revealed in the fact that the characteristic wavelength of such patterns depends both on the parameters of energetic interactions, i.e., on the interaction radius, and on the diffusion length of adsorbed particles. Of course, this physical difference does not prevent similar mathematical methods from being used in theoretical investigations of these different patterns. As shown in the paper, the mathematical stability analysis for the considered systems is, for example, essentially analogous to that performed for the Turing patterns in activator-inhibitor models.

Though theoretical investigations have been performed in this study only for the systems representing adsorbates with surface chemical reactions, they may also be interesting for other systems, such as reactive polymer blends. Indeed, we have effectively considered an example of a system with a first-order phase transition where both reversible equilibrium reactions (i.e., thermal adsorption and desorption) and a nonequilibrium reaction (such as photoinduced desorption) are simultaneously taking place. We have found that, despite the fact that the equilibrium reactions cannot themselves create kinetic microstructures, they significantly influence properties of the microstructures induced by the nonequilibrium reaction.

\section{ACKNOWLEDGMENTS}

The authors acknowledge the help of A. Preusser in numerical simulations on the parallel Cray T3E computer, and thank M. Scheffler for providing access to this facility. 
[1] R. Imbihl and G. Ertl, Chem. Rev. 95, 697 (1995).

[2] S. Jakubith, H. H. Rotermund, W. Engel, A. von Oertzen, and G. Ertl, Phys. Rev. Lett. 65, 3013 (1990).

[3] M. Eiswirth and G. Ertl, in Chemical Waves and Patterns, edited by R. Kapral and K. Showalter (Kluwer, Dordrecht, 1995), p. 447.

[4] J. Wintterlin, S. Völkening, T. V. W. Janssens, T. Zambelli, and G. Ertl, Science 278, 1931 (1997).

[5] A. S. Mikhailov and G. Ertl, Chem. Phys. Lett. 238, 104 (1995); 267, 400 (1997).

[6] A. S. Mikhailov and G. Ertl, Science 272, 1596 (1996).

[7] J. W. Cahn, Acta Metall. 9, 795 (1961).

[8] K. Binder, in Material Science and Technology: Phase Transformations in Materials, edited by P. Haasen (VCH, Weinham, 1990), Vol. 5, p. 405.

[9] S. C. Glotzer, E. A. Di Marzio, and M. Muthukumar, Phys. Rev. Lett. 74, 2034 (1995).

[10] M. Motoyama and T. Ohta, J. Phys. Soc. Jpn. 66, 2715 (1997).

[11] Q. Tran-Cong and A. Harada, Phys. Rev. Lett. 76, 1162 (1996).

[12] J. Verdasca, P. Borckmans, and G. Dewel, Phys. Rev. E 52, R4616 (1995).

[13] M. Hildebrand and A. S. Mikhailov, J. Phys. Chem. 100, 19089 (1996).

[14] R. Lefever, D. Carati, and N. Hassani, Phys. Rev. Lett. 75, 1674 (1995); S. C. Glotzer, D. Stauffer, and N. Jan, ibid. 75, 1675 (1995).

[15] J. W. Cahn and J. E. Hillard, J. Chem. Phys. 28, 258 (1958).

[16] B. Huberman, J. Chem. Phys. 65, 2013 (1976).
[17] See, e.g., J. K. Nørskov, in Coadsorption, Promoters and Poisons, edited by D. A. King and D. P. Woodruff (Elsevier, Amsterdam, 1993), p. 1; T. L. Einstein and J. R. Schrieffer, Phys. Rev. B 7, 3629 (1973); K. H. Lau and W. Kohn, Surf. Sci. 75, 69 (1978).

[18] P. Zeppenfeld, M. Krzyzowski, C. Romainczyk, G. Gomsa, and M. G. Lagally, Phys. Rev. Lett. 72, 2737 (1994); V. I. Marchenko, Pis'ma Zh. Eksp. Teor. Fiz. 55, 72 (1992) [JETP Lett. 55, 73 (1992)]; D. Vanderbilt, Surf. Sci. 268, L300 (1992).

[19] G. Giacomin and J. L. Lebowitz, Phys. Rev. Lett. 76, 1094 (1996).

[20] G. W. Gardiner, Handbook of Stochastic Methods (Springer, Berlin, 1985).

[21] A. S. Mikhailov and A. Yu. Loskutov, Foundations of Synergetics II. Chaos and Noise, 2nd revised ed. (Springer, Berlin, 1996).

[22] T. L. Hill, Statistical Mechanics (McGraw-Hill, New York, 1956).

[23] J. Verdasca, P. Borckmans, and G. Dewel, Phys. Rev. E 55, 4828 (1997).

[24] D. Walgraef, Spatio-Temporal Pattern Formation (Springer, Berlin, 1997).

[25] J. Boissonade, E. Dulos, and P. De Kepper, in Chemical Waves and Patterns, edited by R. Kapral and K. Showalter (Kluwer, Dordrecht, 1995), p. 221; Q. Quyang and H. L. Swinney, ibid., p. 269.

[26] M. Seul and D. Andelman, Science 267, 476 (1995). 